\title{
Seleção da artéria radial para utilização como enxerto aortocoronário: correlação cirúrgica $x$ avaliação pré-operatória com ecocolor Doppler e fotopletismografia digital
}

\author{
Melchior Luis LIMA*, Fábio José dos REIS*, Francisco Otávio Gaburro TEIXEIRA*, \\ Fanilda Souto BARROS*
}

RBCCV 44205-568

Lima M L, Reis F J, Teixeira F O G, Barros F S - Seleção da artéria radial para utilização como enxerto aortocoronário: correlação cirúrgica $x$ avaliação pré-operatória com ecocolor Doppler e fotopletismografia digital. Rev Bras Cir Cardiovasc 2002; 17 (1): 19-23.

Resumo: Objetivo: Com o crescente uso da artéria radial como enxerto aortocoronário, torna-se necessário reavaliar os critérios utilizados para a seleção desse vaso no pré-operatório da cirurgia de revascularização miocárdica. O objetivo deste estudo foi correlacionar a avaliação da viabilidade do vaso pelo cirurgião cardíaco no per-operatório com o estudo de seleção da artéria radial pelos métodos não invasivos, como o ecocolor Doppler e a fotopletismografia digital.

Casuística e Métodos: Foram estudadas, entre julho de 1998 e maio de 2000, 78 artérias radiais e 78 artérias ulnares de 39 pacientes selecionados para cirurgia de revascularização miocárdica. 0 estudo foi realizado bilateralmente, utilizando aparelhos de ultra-sonografia ATL-HDI 5000 e AcusonSequóia 512, e o aparelho de fotopletismografia Parks Vascular Mini-Lab Model 1052-C. A artéria radial foi considerada passível de ser utilizada como enxerto aortocoronário, quando preenchia os seguintes critérios: ausência de ateromatose ou oclusão da artéria radial e ulnar ipsilateral, diâmetro interno da artéria radial maior ou igual a $2,5 \mathrm{~mm}$, avaliados pelo ecocolor Doppler, e presença de onda de pulso em dois ou mais dígitos durante a compressão radial, avaliada pela fotopletismografia.

Resultados: Dentre as 78 artérias radiais estudadas, 64 (82\%) foram consideradas apropriadas para utilização como enxerto aortocoronário e 12 (18,7\%) não apropriadas. Dos 39 pacientes selecionados, $24(61,5 \%)$ foram submetidos à cirurgia de revascularização miocárdica com utilização da artéria radial e a correlação entre a viabilidade da artéria radial avaliada pelo cirurgião durante o per-operatório com a avaliação pré-operatória pelo ecocolor Doppler e pela fotopletismografia para seleção da artéria radial foi de $100 \%$. A contra-indicação da retirada de $12(18,7 \%)$ artérias radiais levou à alteração do planejamento cirúrgico de dois pacientes, devido ao comprometimento bilateral. Em 3 pacientes que, por diferentes motivos, não foram submetidos ao estudo pelo Ecocolor Doppler e pela fotopletismografia, a avaliação clínica da perviedade da artéria radial utilizando a manobra de Allen não foi suficiente para afastar a presença de calcificação da parede arterial encontrada no ato operatório, impossibilitando o uso dessa artéria como enxerto.

Conclusão: Os autores concluem que a seleção da artéria radial para utilização como ponte aortocoronária, avaliada por testes não invasivos, como o ECD e a fotopletismografia, é eficaz, pois a associação desses métodos apresenta excelente correlação com os achados per-operatórios e permite a nós, cirurgiões cardiovasculares, além da segurança, um melhor planejamento cirúrgico para cada paciente.

DESCRITORES: Artéria radial, ultrasonografia. Ecocardiografia Doppler, utilização. Fotoplestimografia, utilização. Revascularização miocárdica.

Trabalho realizado no Instituto de Cardiologia do Espírito Santo. Vitória, ES, Brasil.

Recebido para publicação em agosto de 2000.

*Do Instituto de Cardiologia do Espírito Santo.

Endereço para correspondência: Melchior L. Lima. Rua Alfeu Alves Pereira, 60. Enseada do Suá. Vitória, ES, Brasil. CEP: 29050-190. Tel: (27) 3324-

0304 3227-8654.e-mail: centrocor@centrocor.com.br 
Lima M L, Reis F J, Teixeira F O G, Barros F S - Seleção da artéria radial para utilização como enxerto aortocoronário: correlação cirúrgica x avaliação pré-operatória com Ecocolor doppler e fotopletismografia digital. Rev Bras Cir Cardiovasc 2002; 17 (1): 19-23.

\section{INTRODUÇÃO}

A veia safena interna foi, durante muito tempo, a melhor opção como substituto arterial. Inicialmente descrita por FAVAROLO et al. (1) ainda é utilizada pela maioria dos cirurgiões cardiovasculares como uma alternativa na doença aterosclerótica obstrutiva, mas não a única, nem talvez a melhor. Posteriormente, o uso da artéria mamária (torácica interna) com essa mesma finalidade foi proposto por KOLESSOV et al. ${ }^{(2)}$.

CARPENTIER et al. (3) foram os pioneiros no uso da artéria radial como substituto vascular na cirurgia de revascularização miocárdica, medida inicialmente com resultados ruins, devido ao vasoespasmo peculiar desse vaso. Anos depois, após modificações na técnica cirúrgica e a associação de drogas, tais como bloqueadores de canais de cálcio, com o objetivo de diminuir a incidência de vasoespasmo ${ }^{(4)}$, estimularam a reativação do uso dessa artéria.

A utilização de artéria como enxerto tem se mostrado superior à veia, devido ao calibre semeIhante ao da coronária, à própria constituição da parede e ao maior tempo de patência.

Diante da freqüência do uso da artéria radial como ponte aortocoronária e dos excelentes resultados demonstrados por diversos autores $(4,5)$, é necessário reavaliar os critérios utilizados para a seleção desse vaso no pré-operatório, com o objetivo de estudar a perviedade, a medida do diâmetro, a presença ou ausência de doença aterosclerótica tanto na artéria radial como na ulnar e a avaliação da perfusão digital com manobras que simulem a retirada da artéria radial, evitando, assim, complicações isquêmicas no pós-operatório e possibilitando ao cirurgião informações a respeito do estado anatômico e funcional da artéria em questão.

Relembrando a circulação da mão, sabemos que a irrigação é feita predominantemente pelo arco palmar superficial, que tem a artéria ulnar como principal responsável. Ele é chamado de completo, quando existe continuidade do arco, recebendo suprimento sangüíneo tanto pela artéria radial, como pela ulnar. É considerado incompleto, quando não há ligação entre os ramos terminais de ambas as artérias, de forma que, nesses casos, a retirada da artéria radial poderá levar a prejuízo na perfusão digital.

Segundo COLEMAN et al. ${ }^{(6,7)}$ o arco é completo em $78,5 \%$ da população.

Com o advento do ecocolor Doppler, método diagnóstico vascular não invasivo, tem sido possível selecionar qual segmento venoso ou arterial passível de ser utilizado como substituto arterial (8).
A equipe de cirurgia cardíaca, junto com o grupo de ultra-sonografia vascular, apresenta como objetivo deste trabalho a avaliação da eficácia e utilidade do mapeamento da artéria radial com ecocolor Doppler (ECD) associado à fotopletismografia digital nos pacientes selecionados para a cirurgia de revascularização miocárdica, correlacionando os achados ultrasonográficos com os achados per-operatórios.

\section{CASUÍSTICA E MÉTODOS}

Foram estudadas, no período de julho/1998 a janeiro/2000, 78 artérias radiais e 78 artérias ulnares de 39 pacientes, sendo 27 do sexo masculino e 12 do sexo feminino. A idade variou de 42 a 84 anos.

O mapeamento arterial, incluindo artéria subclávia, braquial, radial e ulnar bilateral com ecocolor Doppler, foi feito utilizando o aparelho de ultra-som ATL HDI 5.000 e Acuson Sequóia 512 com transdutores lineares de $7.5 \mathrm{Mhz}$ e os seguintes parâmetros foram analisados:

1) investigação de doença aterosclerótica na artéria radial e ulnar;

2) medida do diâmetro interno da artéria radial e ulnar pelo ultra-som com o vaso em corte transverso;

3) avaliação da perviedade do vaso pelo Doppler colorido e pulsado. A fotopletismografia foi realizada utilizando o aparelho Parks vascular Mini-Lab Model 1052 - C. Foram obtidas ondas de pulso em todos os dígitos da mão com o paciente sentado, com a mão relaxada durante a realização da manobra de compressão da artéria radial. A artéria radial foi considerada apropriada para a utilização como ponte aortocoronária, quando apresentava os seguintes parâmetros:

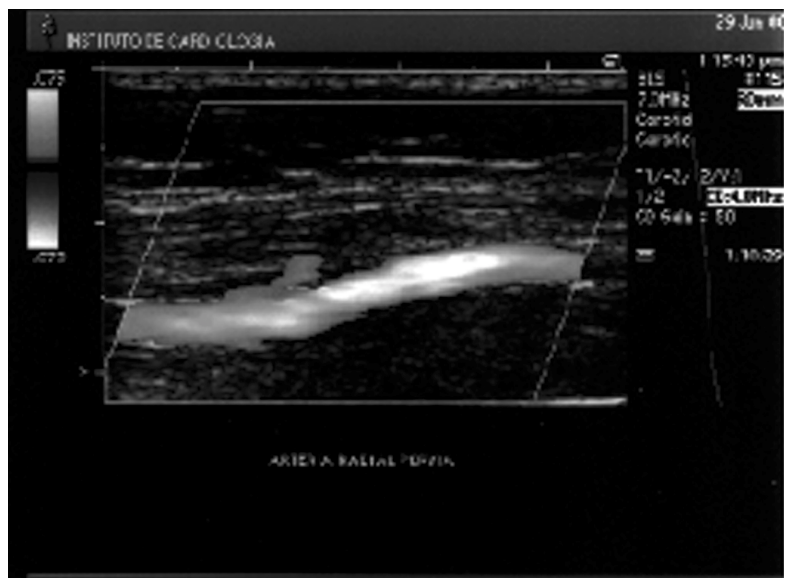

Fig. 1 - Perviedade avaliada pelo ECD perviedade avaliada pelo ECD 
Lima M L, Reis F J, Teixeira F O G, Barros F S - Seleção da artéria radial para utilização como enxerto aortocoronário: correlação cirúrgica x avaliação pré-operatória com Ecocolor doppler e fotopletismografia digital. Rev Bras Cir Cardiovasc 2002; 17 (1): 19-23.

- perviedade avaliada pelo ECD (Figura 1);

- ausência de ateromatose na artéria radial e ulnar, quando avaliada pelo ECD;

- diâmetro interno da artéria radial maior que 2,5mm (Figura 2);

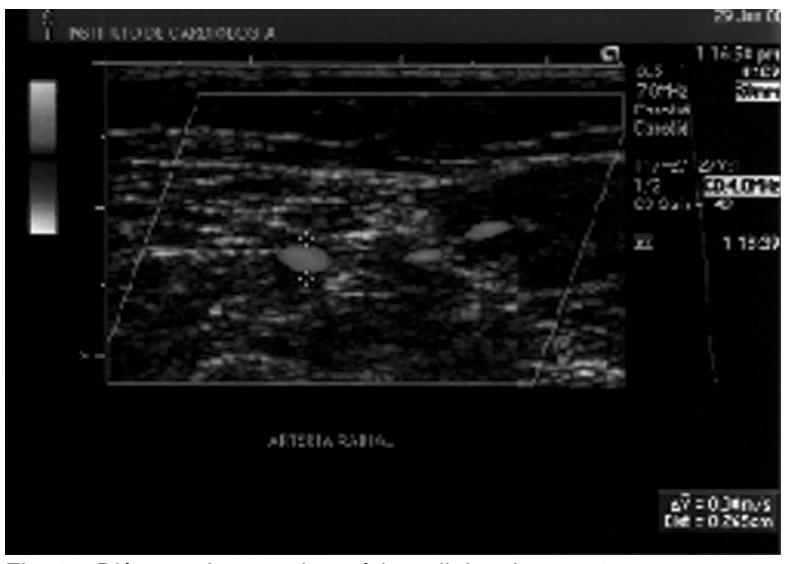

Fig. 2 - Diâmetro interno da artéria radial maior que 2,5mm.

- fotopletismografia demonstrando presença de onda de pulso em todos os dígitos durante a compressão da artéria radial ipsilateral. Os parâmetros avaliados pelo cirurgião cardíaco foram a visibilidade direta da artéria, medida do calibre do vaso utilizando ogivas de diâmetros 1,5 e 3,0mm. Esses dados foram correlacionados com a descrição dos achados ultrasonográficos.

A manobra utilizada pelo cirurgião vascular para avaliação da integridade do arco palmar superficial e posterior correlação com a fotopletismografia consistiu em uma pequena incisão na artéria radial e na avaliação da presença de fluxo retrógrado, que, quando presente, aumentava a segurança de que, após a retirada da artéria radial, a circulação digital se manteria.

\section{RESULTADOS}

Dentre as 78 artérias radiais estudadas em 39 pacientes, 64 (82\%) foram consideradas apropriadas e passíveis de serem retiradas para utilização como enxerto aortocoronário.

Das 64 artérias radiais de 32 pacientes consideradas apropriadas, 24 foram utilizadas como ponte aortocoronária, não havendo discordância entre a avaliação pré-operatória pelos métodos não invasivos e os achados per-operatórios. Foram consideradas não apropriadas 12 artérias (18,7\%). As contra-indicações para a retirada da artéria radial foram devido à:

- presença de doença aterosclerótica da artéria radial evidenciada pelo ECD, em duas;
- presença de doença aterosclerótica da artéria ulnar evidenciada pelo ECD, em três;

- oclusão da artéria braquial evidenciada pelo ECD em duas;

- ausência ou diminuição da onda de pulso em dois ou mais dígitos durante a compressão da artéria radial ipsilateral, avaliadas pela fotopletismografia, em cinco artérias, embora a avaliação pelo ECD demonstrasse artéria radial e ulnar normais, sugerindo, com isso, a presença do arco palmar superficial incompleto.

Em 3 pacientes, nos quais não foi possível a realização da fotopletismografia, o mapeamento da artéria radial e ulnar com o ECD foi suficiente para selecionar a artéria radial para ponte aortocoronária, sendo o achado per-operatório concordante com o resultado do ECD.

Nenhum dos pacientes da nossa casuística apresentou fenômenos isquêmicos no pós-operatório.

\section{COMENTÁRIOS}

Diante do crescente uso da artéria radial como enxerto aortocoronário, do grande avanço tecnológico dos métodos não invasivos para avaliação e seleção de conduto vascular e baseado, em estudos prévios, do nosso grupo junto com o grupo de ultrasonografia vascular, com excelentes resultados entre a correlação cirúrgica e o mapeamento pré-operatório da safena interna com o ECD ${ }^{(9)}$, optamos por desenvolver com a mesma linha de raciocínio, a correlação entre os achados da seleção pré-operatória da artéria radial pelo ECD e pela fotopletismografia digital com a avaliação per-operatória da viabilidade desse vaso como enxerto.

O mapeamento da artéria radial pelo ECD, associado à fotopletismografia digital, tem se mostrado útil na seleção da artéria radial no pré-operatório de cirurgia de revascularização miocárdica, o ECD fornece informações do estado anatômico da artéria e a fotopletismografia digital possibilita uma avaliação indireta da perfusão digital durante a compressão da artéria radial.

KUPINSKI et al. (10) avaliaram, com ECD e fotopletismografia, 146 pacientes antes da cirurgia de revascularização miocárdica, com o objetivo de selecionar a artéria radial para a ponte aortocoronária. Em 22 pacientes de sua casuística, a artéria radial não foi utilizada devido a anormalidades apresentadas pelo ECD, pela fotopletismografia, ou por ambos.

$\mathrm{Na}$ nossa casuística, de 39 pacientes estudados, em $24(61,5 \%)$ a artéria radial foi utilizada como ponte aortocoronária. A contra-indicação da 
Lima M L, Reis F J, Teixeira F O G, Barros F S - Seleção da artéria radial para utilização como enxerto aortocoronário: correlação cirúrgica x avaliação pré-operatória com Ecocolor doppler e fotopletismografia digital. Rev Bras Cir Cardiovasc 2002; 17 (1): 19-23.

retirada de $12(18,7 \%)$ artérias radiais levou à alteração do planejamento cirúrgico de 2 pacientes, devido ao comprometimento bilateral das artérias.

Todos os pacientes de nossa casuística foram interrogados no primeiro mês pós-operatório. Não houve nenhuma reclamação referente à presença de fenômenos isquêmicos.

A correlação per-operatória da qualidade e viabilidade do uso da artéria radial considerada apropriada pela avaliação não invasiva pré-operatória foi de $100 \%$, não havendo resultados falsos negativos. Pelo contrário, em 3 pacientes, que, por diferentes motivos, não foram submetidos ao estudo pelo ECD e pela fotopletismografia, a avaliação clínica da perviedade da artéria pela manobra de Allen não foi suficiente para afastar a presença de calcificação da parede arterial encontrada no ato operatório, impossibilitando, portanto, o uso da artéria como enxerto.

A avaliação pré-operatória do paciente selecionado para operação de revascularização miocárdica, utilizando metodologia não invasiva que possa for- necer informações seguras e confiáveis a respeito do segmento venoso que será utilizado para enxerto aortocoronário, facilita um planejamento cirúrgico mais eficaz, já que podemos saber previamente quais as possibilidades que temos e qual a melhor opção para o paciente, evitando-se, assim, incisões desnecessárias na procura de veia, aumento no tempo cirúrgico e possíveis complicações pós-operatórias inerentes a essas atitudes.

\section{CONCLUSÃO}

Os autores concluem que a seleção da artéria radial para utilização como ponte aortocoronária, avaliada por testes não invasivos, como o ECD e a fotopletismografia, é eficaz, pois a associação desses métodos apresenta excelente correlação com os achados per-operatórios e permite a nós, cirurgiões cardiovasculares, além da segurança, um melhor planejamento cirúrgico para cada paciente.

RBCCV 44205-568

Lima M L, Reis F J, Teixeira F O G, Barros F S - Radial artery selection as coronary artery bypass graft: surgical correlation vs preoperative evaluation by using echocolor Doppler and digital photoplethysmography. Rev Bras Cir Cardiovasc 2002; 17 (1): 19-23.

ABSTRACT: Introduction: With the growing use of the radial artery as a coronary artery bypass graft, becomes necessary to revalue the criteria used for the selection of that vessel in the preoperative of the surgery of coronary artery bypass graft (CABG). The objective of this study was to correlate the evaluation of the viability of the vessel for the heart surgeon at surgery with the study of selection of the radial artery for the methods non invasivos, as EchocolorDoppler(ECD) and the digital photoplethysmography(PPG).

Methods: Between July of 1998 and January of 2000, 78 radial arteries and 78 arteries ulnares of 39 patients selected for CABG, were studied. The study was carried out bilaterally, using duplex ultrasound ATL - HDI 5000 and Acuson - Sequoia 512, and for the photoplethysmography an apparel Parks Vascular Mini - Lab Model 1052-C. The radial artery was considered susceptible to be used as coronary artery graft bypass, when it filled out the following criteria: ateromatose absence or occlusion of the radial artery and ulnar ipsilateral, internal diameter of the larger radial artery or equal to $2.5 \mathrm{~mm}$, appraised for ECD, and presence of pulse wave in two or more digits during the radial compression, appraised for the PPG.

Results: Among the 78 studied radial arteries, 64 (82\%) were considered appropriate for use as coronary artery graft bypass and $12(18.7 \%)$, not appropriate. Of the 39 selected patients, $24(61.5 \%)$ they were submitted to CABG with use of the radial artery and the correlation between the viability of the appraised radial artery for the surgeon at surgery with the evaluation preoperative for ECD and for the PPG for selection of the radial artery was of $100 \%$. The contraindication of the harvest of 12 $(18.7 \%)$ radial arteries took to the alteration of the surgical planning of two patients, owing to the bilateral compromising. In three patients that, for different reasons, they were not submitted to the study by ECD and for the PPG, the clinical evaluation of the patency of the radial artery using Allen's maneuver was not enough to move away the presence of calcification of the arterial wall found in the operative act, disabling the use of that artery as graft.

Conclusion: The selection of the radial artery for use as graft coronary artery bypass, appraised by non invasive tests, as ECD and the PPG, it is effective, because the association of those methods presents excellent correlation with the evaluation at surgery and it allows us, cardiovascular surgeons, besides the safety, a better surgical planning for each patient.

DESCRIPTORS: Radial artery, ultrasonography. Echocardiography, Doppler color, utilization. Photoplethysmography, utilization. Myocardial revascularization. 
Lima M L, Reis F J, Teixeira F O G, Barros F S - Seleção da artéria radial para utilização como enxerto aortocoronário: correlação cirúrgica x avaliação pré-operatória com Ecocolor doppler e fotopletismografia digital. Rev Bras Cir Cardiovasc 2002; 17 (1): 19-23.

\section{REFERÊNCIAS BIBLIOGRÁFICAS}

1 Favarolo R G - Saphenous vein graft in the surgical treatment of coronary artery disease: operative technique. J Thorac Cardiovasc Surg 1969; 58: 178-85.

2 Kolessov V L - Mammary artery-coronary artery anastomosis as a method of treatment for angina pectoris. J Thorac Cardiovasc Surg 1967; 54: 535-44.

3 Carpentier A, Guermonprez J L, Deloche A, Frechette C, DuBost $\mathrm{C}$ - The aorta-to-coronary radial artery bypass graft: a technique avoiding pathological changes in grafts. Ann Thorac Surg 1973; 16: 111-21.

4 Manasse E, Sperti G, Suma $\mathrm{H}$ et al. - Use of the radial artery for myocardial revascularization. Ann Thorac Surg 1996; 62: 1082-3.

5 Da Costa F D, da Costa I A, Poffo R et al. - Myocardial rRevascularization with the radial artery: a clinical and angiografic study. Ann Thorac Surg 1996; 62: 475-80.
6 Yao $\mathrm{J} \&$ Brewster D - Current diagnosis $\mathrm{X}$ treatment in vascular surg; pp 155-7.

7 Winkler J, Lohr J, Hearn A - Evaluation of the radial artery for use in coronary artery bypass grafting. $J$ Vasc Technol 1998; 22: 23-9.

8 Cousens K A, Altemus A P, Musson A M, Zwoulak R M - Utility of properative vein mapping. $J$ Vasc Technol 1997; 21: 227-31.

9 Barros F, Pontes S, Lima M et al. - Mapeamento da safena interna com ecocolor Doppler no pré-operatório de cirurgia de revascularização miocárdica. Rev Bras Cir Cardiovasc 1999; 14: 303-7.

10 Kupinski A, Huang J, Khan A et al. - Noninvasive upper extremity arterial assesment in patients undergoing radial artery harvest. $J$ Vasc Technol 1998; 22: 187-91. 